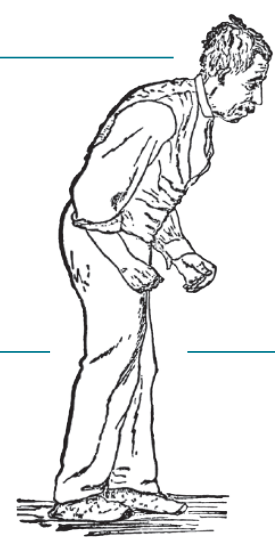

\title{
Two hundred steps
}

\section{7}

James Parkinson, a general practitioner in London, describes six cases of "the shaking palsy" in an early example of defining a neurological condition on the basis of its symptoms. His description of the symptoms and of disease progression (as shown in an illustration from 1886, pictured) remain largely valid today, except for his claim of no cognitive impairment.

Working with Alois Alzheimer in Germany, neurologist Frederic Lewy uses newly developed staining techniques on post-mortem brains of patients who had Parkinson's disease. Inside various groups of subcortical neurons, Lewy finds distinctive protein aggregates (pictured) that will later be named after him. Lewy also finds variability in the occurrence of these aggregates: a few parkinsonian brains lack them, whereas some healthy brains contain them. This starts a debate, that continues to the present day, about the precise relationship between Lewy bodies and Parkinson's disease.

Neuropathologist Konstantin Tretiakoff suggests that the loss of neurons from the brain's substantia nigra is the pathological hallmark of both Parkinson's disease and post-encephalitic

parkinsonism, a similar condition that can occur in people after a viral infection. But other researchers believe that Parkinson's pathology takes root in a different part of the brain called the striatum. It takes decades for Tretiakoff's hypothesis to be accepted.

Oleh Hornykiewicz and Herbert Ehringer at the University of Vienna investigate dopamine levels in the post-mortem brains of two people who had Parkinson's and four who had post-encephalitic parkinson-

ism. In all six, dopamine levels in the striatum are lower than in unaffected brains. A few years later, researchers find that the striatum's dopamine comes from neurons projecting from the substantia nigra.

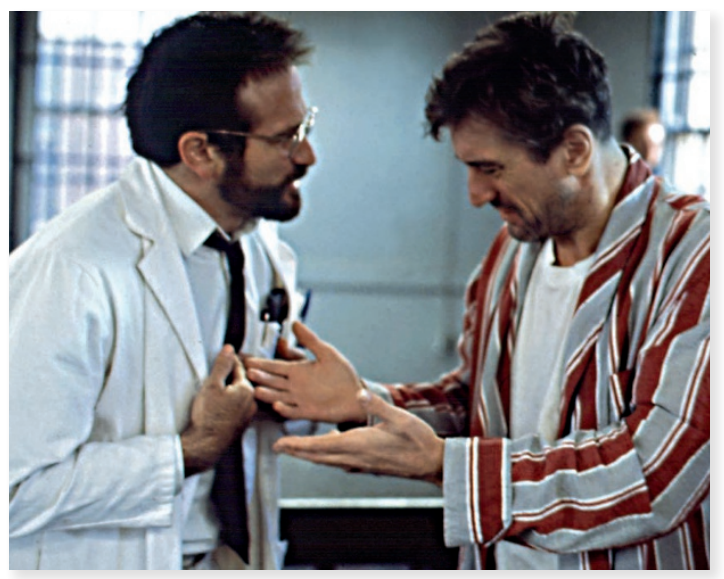

\section{2}

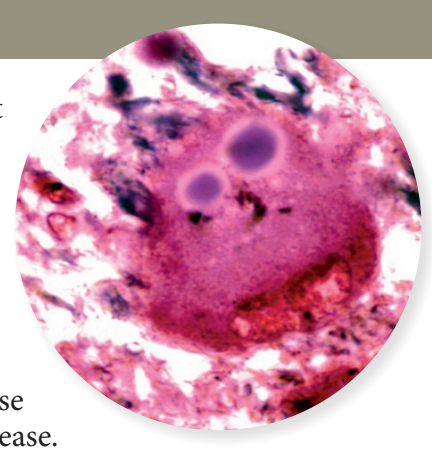

1919

\section{0}

\section{QUOTES FROM THE PAPERS}

"Involuntary tremulous motion, with lessened muscular power ... with a propensity to bend the trunk forwards, and to pass from a walking to a running pace: the senses and intellects being uninjured. " J. Parkinson An Essay on the Shaking Palsy (Sherwood, Neely \& Jones, 1817).

"By better techniques we see one nervous disorder after the other moving on from the functional into the organic level." F. H. Lewy In Lewandowsky's Handbook of Neurology (Springer, 1912).

"The results of our research lead us to think there exist intimate relationships between the substantia nigra and Parkinson's disease. These relations are very likely that of a cause and its effects". K. Tretiakoff. PhD thesis, Univ. Paris (1919).

"Should this finding be confirmed ... a particularly great importance would have to be attributed to dopamine's role in the pathophysiology and symptomology of idiopathic Parkinson's disease and postencephalitic parkinsonism." H. Ehringer \& O. Hornykiewicz Klin. Wochenschr. 38,

1236-1239 (1960), in German.

In a bid to treat Parkinson's by restoring dopamine levels, Hornykiewicz and Walther Birkmayer, also at the University of Vienna, give patients the dopamine precursor levodopa. Within hours, immobile patients can move, and the effects last up to a day. The drug is also successful in people with post-encephalitic parkinsonism (told in the book and film Awakenings, pictured) and is approved in 1970. Other dopaminebased therapies have since been developed, but levodopa is the gold standard for relieving the motor symptoms of Parkinson's.
"Bedridden patients who were unable to sit up, patients who could not stand up from a sitting position, and patients who, when standing, could not start walking, performed all these activities after L-DOPA with ease." W. Birkmayer \& 0 . Hornykiewicz Wien. Klin. Wochenschr. 73, 787-788 (1961). 
In the 200 years since Parkinson's disease was first described, the understanding and management of the disease has come a long way. But researchers have yet to unlock all of its secrets. By Liam Drew.

"We observed high-frequency stimulation ... suppressed the tremor, in a reversible manner, and improved the drawing of figures such as spirals." A. L. Benabid et al. Appl. Neurophysiol. 50, 344-346 (1987).

"Even if the mutation we have described is directly related to only a small fraction of the total number of PD patients, it provides a clue [to] the underlying pathways resulting in symptoms of PD." M. H. Polymeropoulos Science 276, 2045-2047 (1997).

"Parkinson's disease and dementia with Lewy bodies may be $\alpha$-synuclein diseases."

M. G. Spillantini et al. Nature 388, 839-840 (1997).

"This study traces the course of the pathology in incidental and symptomatic Parkinson cases proposing a staging procedure based upon the readily recognizable topographical extent of the lesions." H. Braak et al. Neurobiol. Aging 24, 197-211 (2003).

"There must be either a pathogenic factor in the brain milieu that affects dopaminergic neurons or a pathological process that can spread from one cellular system to another."

J. H. Kordower Nature Med. 14, 504-506 (2008).

"Yet despite the available preclinical and clinical evidence, creatine failed to slow the clinical progression

of Parkinson disease as measured ... in the long-term clinical trial." K Kieburtz J. Am. Med. Assoc. 313, 584-593 (2015).

\section{7}

Neurosurgeon Alim Louis Benabid at Grenoble University in France finds that electrical stimulation of part of the brain's thalamus relieves the motor symptoms of Parkinson's disease (see page S10). Deep brain stimulation becomes popular as a treatment for patients who do not respond to levodopa.

\section{JUNE 1997}

A team led by geneticist Mihael Polymeropoulus at the National Institutes of Health in Bethesda, Maryland, identifies a mutation that causes an inherited form of Parkinson's. The gene produces the protein $\alpha$-synuclein. Duplications and triplications of this gene are later also shown to cause the disease, and mutations in other genes are linked to rare inherited cases.

\section{AUGUST 1997}

Neurobiologists Michel Goedert and Maria Grazia Spillantini are studying a-synuclein at the University of Cambridge, UK, when Polymeropoulus's work comes out. They are intrigued by the Parkinson's connection and devise a study that reveals that the elusive primary constituent of Lewy bodies is a-synuclein.

\section{3}

A group led by neuroanatomist Heiko Braak at Goethe University in Frankfurt, Germany, charts the development of Parkinson's pathology. Braak's team proposes a six-stage process in which lesions and protein aggregates spread through the brain; some of these stages occur before motor symptoms and cell loss. The details are still debated, but 'Braak staging' provides a useful framework for describing the progression of Parkinson's.

\section{8}

Groups from Rush University in Chicago, Illinois, and Lund, Sweden, find that fetal neurons previously transplanted into the brains of people with Parkinson's to restore dopamine production develop Lewy-body-like structures. This suggests that the disease can invade young, healthy tissue. New hypotheses arise, including one suggesting that misfolded $\alpha$-synuclein can spread from cell to cell, much like infectious misfolded proteins called prions (see page S13).

\section{TO PRESENT DAY}

Drugs that halt or slow the progression of Parkinson's disease continue to elude researchers. The NINDS Exploratory Trials in Parkinson Disease Investigators report the latest disappointment: creatine monohydrate. New treatments, some based on genetic insights, are being developed. But James Parkinson's 1817 hope "that some remedial process may ere long be discovered, by which ... the progress of the disease may be stopped" remains to be realized.

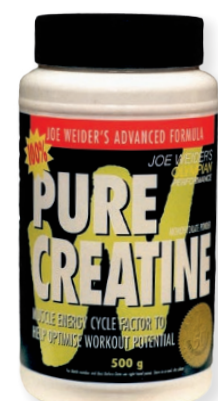

